\title{
Development of a Culturally Tailored Motivational Interviewing-Based Intervention to Improve Medication Adherence in South Asian Patients
}

This article was published in the following Dove Press journal: Patient Preference and Adherence

\author{
Pavneet Singh' \\ Tavis Campbell ${ }^{2}$ \\ Pamela LeBlanc $\mathbb{D}^{1}$ \\ Kathryn M King-Shier $\mathbb{D}^{1,3}$ \\ 'Faculty of Nursing, University of Calgary, \\ Calgary, AB T2N IN4, Canada; \\ ${ }^{2}$ Department of Psychology, University of \\ Calgary, Calgary, AB T2N IN4, Canada; \\ ${ }^{3}$ Department of Community Health \\ Sciences, University of Calgary, Cumming \\ School of Medicine, University of Calgary, \\ Calgary, AB, Canada
}

Background: South Asians (SAs) are among the fastest growing ethnic population in Western countries and have a higher risk of cardiovascular diseases relative to the general population. SAs living in Canada also have poorer adherence to medical regimens for treating cardiovascular disease, relative to other ethnic groups. Motivational interviewing (MI) maybe effective in improving health-related behaviour change in patients; however, the research is nascent on the effectiveness of MI in SAs and may also require cultural adaptation.

Aim: To develop a culturally tailored MI-based intervention to improve medication adherence in hypertensive SA patients living in Canada.

Methods: Previous literature about medication adherence in SAs was reviewed, along with transcripts and responses to open-ended survey questions from our previous studies with SAs, to draft an MI intervention manual. The manual received extensive feedback from the study team, SA community members and health-care providers who work with SA patients. The feedback was used to refine the manual and make it culturally tailored and relevant to SA hypertensive patients living in Canada.

Results: A culturally tailored MI-based manual which we called a "motivational communication manual" was developed to support a study focused on improving medication adherence in SA hypertensive patients. The development process, components (eg, being culturally sensitive, family involvement, providing education about medications, reminders, etc.) and cultural nuances included in the manual are described in this paper.

Conclusion: This is the first culturally tailored MI-based intervention manual that has been developed with the aim of improving medication adherence in hypertensive SA patients and that includes direct feedback from SA community members. Use of this manual may improve the accessibility and adoption of MI-based practices in improving health behaviours in SAs living in Canada as well as encourage further research studies and clinical trials with SA patients.

Keywords: motivational interviewing, medication adherence, South Asians, ethnicity, hypertension

\section{Introduction}

South Asians (SAs; Indian, Pakistani, Bangladeshi, Nepali, Sri Lankan) are among Canada's largest and most rapidly growing ethnic groups, accounting for approximately $25 \%$ of the total visible minority population and $5 \%$ of Canada's total population. ${ }^{1}$ Visible minorities in Canada are described as "persons, other than Aboriginal peoples, who are non-Caucasian in race or non-white in colour", and
Correspondence: Kathryn M King-Shier Faculty of Nursing, University of Calgary, 2500 University Drive NW, Calgary, AB T2N IN4, Canada

Email kingk@ucalgary.ca 
consist mainly of South Asian, Chinese, Black, Filipino, Latin American. Cardiovascular disease, to which coronary artery disease (CAD) is a major contributor, is arguably one of SA's most pressing chronic diseases worldwide. $^{2-4}$ Persons of SA origin have greater morbidity from CAD and typically develop CAD approximately 10 years earlier than their white counterparts. ${ }^{5-8}$

SAs' management of chronic CAD is also worse than the general population. SAs are less likely to follow a heart-healthy diet, participate in exercise programs, or achieve glucose, blood pressure and lipid targets. ${ }^{9-11} \mathrm{SA}$ cardiac patients also have significant challenges with medication adherence. Studies have suggested poor adoption of heart-healthy behaviours such as medication adherence by SAs, ${ }^{12,13}$ with $30 \%$ of SAs being non-adherent to cardiac medications compared to $15 \%$ in the general population. ${ }^{14}$ Studies have also identified specific barriers that may contribute to medication non-adherence (eg, medication side-effects, cost, forgetfulness, higher frequency of dosing, language-barriers) in this group, ${ }^{2-4}$ although these barriers may also apply to the general population. $^{15}$ Therefore, a patient-centred, culturally tailored approach to chronic CAD management that improves motivation to engage in heart-healthy behaviours is needed to close this substantial gap. Although not all barriers are motivational in nature, improving a patient's motivation and invoking their reasons for change can help them prioritize heart-healthy behaviours.

Motivational interviewing (MI) is a collaborative, person-centred form of guiding to elicit and strengthen motivation for change by evoking an individual's own reasons for change, working through ambivalence and perceived barriers to behaviour modifications, as well as fostering selfefficacy. ${ }^{16}$ The key MI strategies to develop discrepancy and evoke commitment to change include the following: 1) asking permission (soliciting patient's permission before sharing information), 2) reflective listening, 3) using openended questions, 4) eliciting response, 5) providing affirmations, 6) working with a scale (a rating scale used to help quantify the patient's readiness to improve their condition) and 7) summarizing. MI has been shown to be effective in enhancing health-behaviour change in several patient populations, including patients with coronary risk factors. ${ }^{17} \mathrm{~A}$ review of 72 clinical trials using $\mathrm{MI}$ suggests that $\mathrm{MI}$ is more effective with minority populations than it was with white populations, ${ }^{18}$ although the reasons for this were not determined. In addition, a recent review ${ }^{19}$ showed that MI, especially when culturally adapted is generally effective in promoting behaviour change in ethnic groups. Culturally adapted interventions are tailored to patients' norms, beliefs, values, language and literacy skills. ${ }^{20,21}$ Most ethnic participants in the included studies also reported acceptance of MI-based interventions. Only one study examined MI in SA patients and revealed that MI was an effective intervention in enhancing smoking cessation. ${ }^{22}$ MI has never been examined in SA cardiac patients, thus, suggesting a strong need to examine the potential effectiveness and acceptability of MI in the SA cardiac population, especially when the intervention is culturally adapted.

The aim of this project was to work in partnership with SA participants as well as health-care providers who work with SA patients to develop a culturally sensitive MIbased intervention manual to improve medication adherence in hypertensive SA patients. We chose hypertensive patients because hypertension is a major risk factor for cardiovascular disease. SAs also have high incidence and poor control of hypertension. ${ }^{23}$ In addition, SAs are known to experience greater side-effects (especially cough) of blood pressure medications compared to people of other ethnicities. ${ }^{24}$ In this paper, we describe how the MI-based intervention manual was developed and adapted to the cultural nuances of SA hypertension patients based on existing literature and qualitative data collected by our research group during previous studies with SAs.

\section{Methods}

\section{Manual Development}

The initial version of the manual was developed by first reviewing the existing literature on medication adherence in SAs. ${ }^{25-27}$ In addition, previous qualitative data collected during hundreds of hours of interviews for research studies conducted by our group (PhD-trained researchers, including a psychologist and nurse, as well as a SA community member) with SA cardiac patients were used in developing and culturally tailoring this manual. ${ }^{28}$ We interrogated previously collected qualitative interviews $(n=64)$, focus groups $(n=18$ participants) and responses to open-ended survey questions $(n=900)$ from SA cardiac patients to identify potential motivators and detractors of medication adherence. ${ }^{13,34}$ These motivators and detractors are listed in Table 1.

We used the literature, data from previous studies (as identified above), as well as feedback received from our research group regarding individual motivators and detractors and the best ways of incorporating them into an intervention manual to draft a culturally tailored MI-based 
Table I Potential Motivators and Detractors for Medication Adherence in South Asians

\begin{tabular}{|c|c|}
\hline Motivators & Detractors \\
\hline Obeying the doctor & $\begin{array}{l}\text { Poor relationship with doctor } \\
\text { (poor communication) }\end{array}$ \\
\hline Staying well for family & Lack of trust in medication \\
\hline Religious obligation & $\begin{array}{l}\text { Sense of fatalism (God has put } \\
\text { this in my hands) }\end{array}$ \\
\hline Understanding the medication & Fear of medications \\
\hline Having money/insurance & Medication side effects \\
\hline $\begin{array}{l}\text { Keeping time on "my side" (I am } \\
\text { so young) }\end{array}$ & $\begin{array}{l}\text { Lack of trust of health-care } \\
\text { providers }\end{array}$ \\
\hline $\begin{array}{l}\text { Staying healthy and avoiding } \\
\text { future problems }\end{array}$ & $\begin{array}{l}\text { Inconvenience/busy schedule } \\
\text { (too much work to remember) }\end{array}$ \\
\hline $\begin{array}{l}\text { Having a positive relationship } \\
\text { with the health-care providers }\end{array}$ & $\begin{array}{l}\text { Absence of knowing if medication } \\
\text { is missed (ie, not noticed) }\end{array}$ \\
\hline $\begin{array}{l}\text { Ease of use } \\
\text { Fear of another event }\end{array}$ & Not getting expected benefits \\
\hline
\end{tabular}

manual. In addition, we propose that "time orientation" is an aspect of health behaviour among SA patients and was used as a guide to develop the manual. Time orientation is a cultural preference toward past, present, or future thinking. Time orientation affects the way a culture values time and the extent to which people believe they can control time. ${ }^{35}$ The manual also included components of the COM-B model of behaviour change ${ }^{17}$ and the Four Habits model. ${ }^{18}$ The COM-B model recognizes that behaviour is based on the interaction of capability, opportunity, motivation and behaviour (COM-B). ${ }^{36}$ According to the COM-B model, behaviour change interventions must change one or more of the components of COM-B. The Four Habits model describes effective medical interviewing skills and its interdependent components are: (1) invest in the beginning, (2) elicit the patient's perspective, (3) demonstrate empathy and (4) invest in the end. ${ }^{37}$

\section{Seeking Feedback to Refine the Manual}

After creating the first draft of the manual, it was sent out to the study team to receive extensive feedback regarding its pragmatic value and cultural-acceptability of the approach. We also received feedback on the motivators and detractors from members of the SA community members $(n=9)$ and family physicians $(n=3)$ who worked with SA patients. A sample feedback form is shown in Figure 1. Based on the feedback received, the manual was further refined after which it was sent out to family physicians $(n=3)$ who work with SA patients to receive extensive feedback.
Inclusion criteria for SA community members (non-healthcare professionals) included: adult ( $\geq 18$ years), communitydwelling man or woman who self-reported their ethnicity as SA; who spoke English, Punjabi or Hindi (the most frequently spoken SA languages in Canada ${ }^{1}$ ). Inclusion criteria for health-care professionals included: community health professional who worked with SA patients and who spoke English, Punjabi or Hindi. The manual was further refined using the feedback received to make it appropriate and culturally tailored in order to improve medication adherence in hypertensive SA patients.

Since there were no participants enrolled for the manual development process, approval by an institutional review board or ethics committee was not required.

\section{Results}

A culturally tailored MI-based manual which we titled the "motivational communication (MC) manual" was developed to address the effectiveness of MI-based approaches for improving medication adherence in SA hypertension patients. Based on the feedback we received, many cultural nuances were included in the intervention manual which are described below.

\section{Cultural Nuances Incorporated into the Manual}

SA culture is generally a "collectivist culture" rather than an "individualistic culture" and family and community is considered a very important part in the life of SAs. ${ }^{29,38,39}$ Central to the SA culture is the desire and commitment to be there for and care for their families (parents, grandparents, children, close relatives). ${ }^{38}$ Often, family members are involved in SA patients' health management and play a role in the patient's decision to take their medications. ${ }^{29}$ Supportive family members have also been shown to improve medication adherence in SA patients by encouraging and reminding patients to take their medications, translating medical information for them and providing them transport to the pharmacy/getting medications for them. ${ }^{29,38,39}$ Therefore, it is helpful to become familiar with the participant's unique family history and responsibilities. Not only can this build rapport, it can also be used to elicit confidence/change talk by invoking family reasons for taking medications. In a similar way, becoming familiar with the participant's community responsibilities and the role of community in their life and 
Background: From our previous studies, we identified several factors which could act as motivators or detractors for South Asian patients to adhering to cardiac medications. After receiving feedback regarding the 'cultural acceptability' of the identified factors from South Asians and healthcare professionals who work with South Asians, we aim to incorporate these factors into motivational communication strategies to tailor them specifically for enhancing medication adherence among South Asians.

Instructions: In the space provided below each item, please provide your feedback regarding the cultural acceptability of including the following motivators and detractors into motivational communication strategies for enhancing South Asians' medication adherence. If required, there is additional space at the end to provide general/overall feedback.

MOTIVATORS:

1) Obeying the doctor

2) Staying well for family

3) Religious obligation

4) Understanding the medication

5) Having money/insurance

6) Keeping time on 'my side' (I'm so young)

7) Staying healthy and avoiding future problems

8) Having a positive relationship with the healthcare providers

9) Ease of use

10) Fear of another event

\section{DETRACTORS:}

1) Medication side effects

2) Poor relationship with doctor (poor communication)

3) Lack of trust of healthcare providers

4) Lack of trust in medication

Figure I Continued. 
5) Sense of fatalism (God has put this in my hands)

6) Fear of medications

7) Inconvenience/busy schedule (too much work to remember)

8) Absence of knowing if medication is missed (i.e., not noticed)

9) Not getting expected benefits

Additional Comments/Notes:

Figure I Feedback form to test the cultural acceptability of motivators and detractors to medication adherence.

use this information to steer the participant towards change talk.

SAs have been shown to have lower understanding of cardiovascular diseases and of the medications prescribed to treat them. ${ }^{25} \mathrm{SA}$ patients' medication adherence is enhanced when they have a better understanding and knowledge about their medications. ${ }^{26,32,33,40}$ Therefore, the role of increasing patient's knowledge can be crucial to improving medication adherence. This is achieved in the manual by testing patient's knowledge about their medications and providing them general information (as needed) about their medications in a patient-centred manner.

SAs also tend to have a present-fatalistic orientation (what one does today would not necessarily influence any future outcomes) rather than a future orientation that is often associated with health-care provision (eg, if you do this today, then that will happen tomorrow) (unpublished data). Thus, it is important that rather than examining the problems their condition has caused in the past or the future benefits of taking medications as prescribed, more emphasis is placed on the present consequences of their behaviour. For example, invoking a desire to care for their family or being there for their grandchildren in the present could potentially lead to a positive behaviour change in SA patients.

Use of a culturally sensitive greeting (Hello, Namaste, Sat Sri Akal, etc.) when meeting with the SA participant was also suggested in order to build rapport with patients. Handshakes, especially with older participants were suggested only when initiated by the participant. Further, initiating a handshake with participants of the opposite sex were also cautioned, unless initiated by the participant. Moreover, religion can be an important part in the lives of many SAs ${ }^{6,7}$. Therefore, it is important to ask participants about the impact of their religion in influencing their decision to take their medications. The response can be used by the interviewer to further guide change talk. An example of a question which can be used by the researcher using a 5-point Likert scale is described in Box 1.

SAs often tend to use traditional herbal remedies along with their medications, and in some cases, prefer them 
Box I General Recommendation for Using MI-Based Approaches with Hypertensive South Asian Patients

- Use greeting to build rapport. Use a culturally sensitive greeting (Hello, Namaste, Sat Sri Akal, etc.) when meeting with the participant. Handshake (especially with older participants) only if the participant initiates. However, avoid handshakes with participants of opposite sex unless the participant extends the hand themselves. Sustained eye contact is also not recommended.

- It is important to understand the participant's concerns about medications rather than explaining the benefits of the medications to them. Some particular concerns may be: It is helpful to acknowledge that it is normal to have concerns/fears/apprehensions regarding medications.

- Regularly test participant comprehension by asking them to repeat what the interviewer has summarized. Example: their reasons for taking medications, their motivators and the steps they will take to adhere to their medications.

- Also, before offering any suggestions, ask permission first. Only proceed if the participant says yes.

- Note: Only discuss blood pressure medications. It is okay for the participants to talk about other medications that they are currently taking or took in the past. However, only use this information to identify the participant's motivators and detractors and use the information for reflections during the session. However, do not go into detail into other medications. If the participant starts talking about different medications, redirect them towards the target behaviour. Example: Perhaps it would be more beneficial to you to talk about $\mathrm{XYZ}$ medications with your doctor or pharmacist as they can provide you better information on them.

- If the participant agrees to proceed, thank them. Ask them if they have any questions before starting.

- Discuss treatment goals and express respect towards alternative healing practices, as these can be culturally driven.

- Compliment participants on efforts to adhere to their medications.

- Use open-ended questions to explore the participant's perspective.

- The interviewer's role in goal setting is to facilitate the participant's planning and problem-solving by asking about strategies that the participant thinks may be helpful. Although the interviewer may ask guiding questions (eg, "What has worked for you in the past?"), no direct advice regarding strategies for change would be provided by the interviewer without participant's prior permission. In addition, the interviewer will not talk about medication dose, etc. Rather, the interviewer will ask the participant to talk with their doctor or pharmacist if they have medication-specific questions, should it come up in the interview.

- When appropriate, use a 5-point Likert scale to examine how various factors can affect patient's desire/ability to adhere to their medications. For example:

Do religious obligations and beliefs influence your desire/decision to take your medications as prescribed?

\begin{tabular}{|c|c|c|c|c|}
\hline 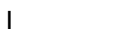 & 2 & 3 & 4 & 5 \\
\hline Not at all & Not much & Neutral/Undecided & Somewhat & Very much \\
\hline \multicolumn{5}{|c|}{$\begin{array}{l}\text { If the answer is either } 4 \text { or } 5 \text {, a follow up question can be asked by the } \\
\text { researcher. For example, we asked: In what ways can your high blood } \\
\text { pressure affect your ability to fulfil your religious obligations and duties in } \\
\text { the future? }\end{array}$} \\
\hline
\end{tabular}

over the medications they have been prescribed. ${ }^{27}$ SAs also experience greater side-effects (especially cough) of blood pressure medications compared to people of other ethnicities, which can contribute to non-adherence. ${ }^{24}$ Therefore, understanding patients' concerns and asking them how they can best manage the conflict between traditional medicines and Western medicines, especially when they experience medication side effects could contribute to improved medication adherence.

Convenience of and having reminders to take medications can also play a critical role in enhancing medication adherence. SAs often prefer to get their medication from pharmacies which are conveniently located for them to visit, where the pharmacist can speak to them in the same language as theirs and remain non-judgmental regarding their non-adherence. ${ }^{29}$ Setting reminders (eg, taking medicines after shower, praying, etc.) and writing schedule on the medication vials in their native language has also been shown to help SA patients adhere to their medications better. ${ }^{29}$ These considerations were included in the form of suggestions which can be provided to SA patients if their lower rate of medication adherence is due to these factors. Some general recommendations for using MI-based approaches with hypertensive SA patients were also included in the intervention manual (Box 1).

\section{Discussion}

We developed a culturally sensitive motivational communication manual for improving medication adherence in SA patients. The intervention manual is available upon request following publication. Constructing the motivational communication manual involved incorporating components from previous research ${ }^{25}$ as well as extensive feedback from the study team, SA community members and healthcare providers who work with SA patients. It is important to note that this manual is intended to be used as a guideline rather than a script, emphasizing maintenance of the spirit of MI during the sessions rather than using rote questions and responses, while still being culturally sensitive. In particular, it seems important to not assume stereotypes of SAs, but rather to approach each person as an individual with their own set of beliefs and practices.

Some previous studies have determined that the effectiveness of MI-based interventions is enhanced when the cultural differences of the participants are acknowledged and the intervention is culturally adapted. ${ }^{19,41-45}$ The one study which examined MI in SA populations did not use a 
culturally tailored MI intervention, even though the study yielded positive results after an MI intervention. ${ }^{22}$ While a pure MI-based approach may work in SA patients, we speculate that making the components of the MI-based approach more culturally tailored could have an enhanced effect on the acceptability of the intervention among SA patients and may even increase the effectiveness of the MI-based intervention.

This motivational communication manual provides guidelines to researchers, counselors and health-care providers to apply $\mathrm{MI}$ in a culturally appropriate manner to SA patients. Care should be taken, however, to avoid cultural stereotypes and proceeding with attention to individual differences. Scientific evaluations of the effectiveness of this adapted version of MI-based intervention will provide more guidance for further adaptations for SA and other diverse populations.

Although this manual has been tailored to hypertensive patients, we believe that the components of this manual can be applied to improving medication adherence for other related conditions in SA patients (diabetes, hypercholesterolemia, etc.). This is because we expect the drivers of medication adherence to be relatively consistent across different conditions. Our research team is currently conducting a pilot study examining the efficacy of this MC manual in improving medication adherence in hypertensive SA patients. Further work needs to be done to develop a comprehensive understanding on the effectiveness of culturally tailored MI-based interventions in SA patients. As SAs have a greater risk of CAD compared to the general population along with a poor management of $\mathrm{CAD}$, this manual has the potential to contribute towards improving health outcomes in SA patients with CAD.

\section{Limitations}

Though this is the first MI-based intervention manual developed for hypertensive SA patients to improve their medication adherence, there are a few potential limitations. As this manual is developed specifically for hypertension, it may not be completely applicable to other health conditions without modifications. It is also possible that this culturally tailored manual may not be completely relevant to second and third-generation SAs in western countries. The manual may also not be applicable to all SA patients as SAs (like most all ethnic groups) are a diverse group of people with variability in their cultures. In addition, as our pilot test is ongoing, the efficacy of this intervention manual has not been established yet. In addition, further changes to this manual are possible based on the feedback received from participants in the pilot study.

\section{Conclusion}

A culturally tailored MC manual developed using direct feedback from SA community members and health-care providers who work with SA patients can be adapted and used to improve health behaviours and conducting research studies with SAs while being culturally sensitive.

\section{Acknowledgments}

This work was funded through the Guru Nanak Dev Ji DIL Research Chair held by KKS.

\section{Disclosure}

There is no conflict of interest to declare.

\section{References}

1. Statistics Canada. Immigration and ethnocultural diversity in Canada. Available from: https://www12.statcan.gc.ca/nhs-enm/2011/as-sa/99010-x/99-010-x2011001-eng.cfm. Accessed July 23, 2019.

2. Yusuf S, Hawken S, Ounpuu S, et al. Effect of potentially modifiable risk factors associated with myocardial infarction in 52 countries (the INTERHEART study): case-control study. Lancet. 2004;364(9438): 937-952.

3. Yusuf S, Reddy S, Ounpuu S, Anand S. Global burden of cardiovascular diseases: part II: variations in cardiovascular disease by specific ethnic groups and geographic regions and prevention strategies. Circulation. 2001;104(23):2855-2864.

4. Bhopal R, Unwin N, White M, et al. Heterogeneity of coronary heart disease risk factors in Indian, Pakistani, Bangladeshi, and European origin populations: cross sectional study. BMJ. 1999;319(7204):215220.

5. Zaman MJS, Philipson P, Chen RL, et al. South Asians and coronary disease: is there discordance between effects on incidence and prognosis? Heart. 2013;99(10):729-736.

6. Khan NA, Grubisic M, Hemmelgarn B, Humphries K, King KM, Quan HD. outcomes after acute myocardial infarction in South Asian, Chinese, and white patients. Circulation. 2010;122(16):1570-1577.

7. Anand SS, Yusuf S, Vuksan V, et al. Differences in risk factors, atherosclerosis, and cardiovascular disease between ethnic groups in Canada: the Study of Health Assessment and Risk in Ethnic groups (SHARE). Lancet. 2000;356(9226):279-284.

8. Sheth T, Nair C, Nargundkar M, Anand S, Yusuf S. Cardiovascular and cancer mortality among Canadians of European, south Asian and Chinese origin from 1979 to 1993: an analysis of 1.2 million deaths. Can Med Assoc J. 1999;161(2):132-138.

9. Liu R, So L, Mohan S, Khan N, King K, Quan H. Cardiovascular risk factors in ethnic populations within Canada: results from national cross-sectional surveys. Open Med. 2010;4(3):e143-e153.

10. Gray J, Millett C, Saxena S, Netuveli G, Khunti K, Majeed A. Ethnicity and quality of diabetes care in a health system with universal coverage: population-based cross-sectional survey in primary care. J Gen Intern Med. 2007;22(9):1317-1320.

11. Astin F, Atkin K, Darr A. Family support and cardiac rehabilitation: A comparative study of the experiences of South Asian and WhiteEuropean patients and their carer's living in the United Kingdom. Eur J Cardiovasc Nur. 2008;7(1):43-51. 
12. Chong E, Wang H, King-Shier KM, Quan H, Rabi DM, Khan NA. Prescribing patterns and adherence to medication among South-Asian, Chinese and white people with Type 2 diabetes mellitus: a populationbased cohort study. Diabetic Med. 2014;31(12):1586-1593.

13. King-Shier K, Quan HD, Mather C, Chong E, LeBlanc P, Khan N. Understanding ethno-cultural differences in cardiac medication adherence behavior: a Canadian study. Patient Prefer Adherence. 2018;12:1737-1747.

14. Lai EJ, Grubisic M, Palepu A, Quan HD, King KM, Khan NA. Cardiac medication prescribing and adherence after acute myocardial infarction in Chinese and South Asian Canadian patients. BMC Cardiovasc Disord. 2011;11:56.

15. Yap AF, Thirumoorthy T, Kwan YH. Systematic review of the barriers affecting medication adherence in older adults. Geriatr Gerontol Int. 2016;16(10):1093-1101.

16. Rollnick S, Miller WR, Butler CC. Motivational Interviewing in Health Care: Helping Patients Change Behavior. New York, NY, US: Guilford Press; 2008.

17. Thompson DR, Chair SY, Chan SW, Astin F, Davidson PM, Ski CF. Motivational interviewing: a useful approach to improving cardiovascular health? J Clin Nurs. 2011;20(9-10):1236-1244.

18. Hettema J, Steele J, WR M. Motivational interviewing. Annu Rev Clin Psychol. 2005;1:91-111. (1548-5943 (Print)).

19. Bahafzallah L, Hayden KA, Raffin-Bouchal S, Singh P, King-Shier KM. Motivational interviewing in ethnic populations. J Immunol Min Health (In Review). 2019.

20. Pottie K, Hadi A, Chen J, Welch V, Hawthorne K. Realist review to understand the efficacy of culturally appropriate diabetes education programmes. Diabet Med. 2013;30(9):1017-1025.

21. Hodge DR, Jackson KF, Vaughn MG. Culturally sensitive interventions and health and behavioral health youth outcomes: a metaanalytic review. Soc Work Health Care. 2010;49(5):401-423.

22. Mahajan R, Solanki J, Kurdekar RS, Gupta S, Modh A, Yadav O. Educating the handicraft factory workers about tobacco cessation and to assess its effectiveness by motivational interviewing: an intervention study. $J$ Exp Ther Oncol. 2017;12(1):43-49.

23. Prabhakaran D, Jeemon P, Ghosh S, et al. Prevalence and incidence of hypertension: results from a representative cohort of over 16,000 adults in three cities of South Asia. Indian Heart J. 2017;69(4):434-441.

24. McDowell SE, Coleman JJ, Ferner RE. Systematic review and metaanalysis of ethnic differences in risks of adverse reactions to drugs used in cardiovascular medicine. BMJ. 2006;332(7551):1177-1181.

25. Hashmi SK, Afridi MB, Abbas K, et al. Factors associated with adherence to anti-hypertensive treatment in Pakistan. PLoS One. 2007;2(3):e280.

26. Patel M, Phillips-Caesar E, Boutin-Foster C. Barriers to lifestyle behavioral change in migrant South Asian populations. J Immigr Minor Health. 2012;14(5):774-785.

27. Kumar K, Greenfield S, Raza K, Gill P, Stack R. Understanding adherence-related beliefs about medicine amongst patients of South Asian origin with diabetes and cardiovascular disease patients: a qualitative synthesis. BMC Endocr Disord. 2016;16(1):24.

28. King KM, Khan N, LeBlanc P, Quan HD. Examining and establishing translational and conceptual equivalence of survey questionnaires for a multi-ethnic, multi-language study. J Adv Nurs. 2011;67(10): $2267-2274$.
29. Ens TA, Seneviratne CC, Jones C, King-Shier KM. Factors influencing medication adherence in South Asian people with cardiac disorders: an ethnographic study. Int J Nurs Stud. 2014;51(11):14721481.

30. King-Shier KM, Singh S, LeBlanc P, et al. The influence of ethnicity and gender on navigating an acute coronary syndrome event. Eur $J$ Cardiovasc Nurs. 2015;14(3):240-247.

31. Singh P, Hayden KA, Ens T, et al. ethno-cultural preferences in receipt of heart health information. Am J Health Behav. 2017;41 (2):114-126.

32. Ens TA, Seneviratne CC, Jones C, Green TL, King-Shier KM. South Asians' cardiac medication adherence. Eur J Cardiovasc Nurs. 2014; 13(4):357-368.

33. King-Shier KM, Singh S, Khan NA, et al. Ethno-cultural considerations in cardiac patients' medication adherence. Clin Nurs Res. 2017;26(5):576-591.

34. King-Shier K, Lau A, Fung S, LeBlanc P, Johal S. Ethnocultural influences in how people prefer to obtain and receive health information. J Clin Nurs. 2018;27(7-8):e1519-e1528.

35. Zimbardo PG, Boyd JN. Putting time in perspective: a valid, reliable individual-differences metric. In: M. Stolarski, N. Fieulaine, W. van Beek, editors. Time perspective theory; Review, research and application: Essays in honor of Philip G. Zimbardo. Springer International Publishing; 2015:17-55.

36. Michie S, van Stralen MM, West R. The behaviour change wheel: a new method for characterising and designing behaviour change interventions. Implement Sci. 2011;6.

37. Frankel RM, Stein T. Getting the most out of the clinical encounter: the four habits model. Perm J. 1999;3(3):79-88. (8755-0229 (Print)).

38. Ahmed SM, Lemkau JP. Cultural issues in the primary care of South Asians. J Immigr Health. 2000;2(2):89-96.

39. Galanti GA. Caring for Patients from different cultures by Geri-Ann Galanti. J Hosp Librariansh. 2015;15(3):341-342.

40. Saleem F, Hassali MA, Shafie AA, Awad AG, Bashir S. Association between knowledge and drug adherence in patients with hypertension in Quetta, Pakistan. Trop J Pharm Res. 2011;10(2):125-132.

41. Longshore D, Grills C, Annon K. Effects of a culturally congruent intervention on cognitive factors related to drug-use recovery. Subst Use Misuse. 1999;34(9):1223-1241.

42. Lee CS, Lopez SR, Colby SM, et al. Culturally adapted motivational interviewing for Latino heavy drinkers: results from a randomized clinical trial. J Ethn Subst Abuse. 2013;12(4):356-373.

43. Hosseini N, Mokhtari S, Momeni E, Vossoughi M, Barekatian M. Effect of motivational interviewing on quality of life in patients with epilepsy. Epilepsy Behav. 2016;55:70-74.

44. Field C, Caetano R. The role of ethnic matching between patient and provider on the effectiveness of brief alcohol interventions with Hispanics. Alcohol Clin Exp Res. 2010;34(2):262-271.

45. Interian A, Lewis-Fernandez R, Gara MA, Escobar JI. A randomizedcontrolled trial of an intervention to improve antidepressant adherence among Latinos with depression. Depress Anxiety. 2013;30(7):6 $88-696$. 


\section{Publish your work in this journal}

Patient Preference and Adherence is an international, peer-reviewed, open access journal that focuses on the growing importance of patient preference and adherence throughout the therapeutic continuum. Patient satisfaction, acceptability, quality of life, compliance, persistence and their role in developing new therapeutic modalities and compounds to optimize clinical outcomes for existing disease

states are major areas of interest for the journal. This journal has been accepted for indexing on PubMed Central. The manuscript management system is completely online and includes a very quick and fair peer-review system, which is all easy to use. Visit http:// www.dovepress.com/testimonials.php to read real quotes from published authors. 